\title{
Quelle recherche en histoire des sciences et des techniques en IUFM?
}

\section{Alain Bernard et Sylvain Laubé}

\section{(2) OpenEdition \\ Journals}

Édition électronique

URL : http://journals.openedition.org/trema/92

DOI : 10.4000/trema.92

ISSN : 2107-0997

Éditeur

Faculté d'Éducation de l'université de Montpellier

Édition imprimée

Date de publication : 1 octobre 2006

Pagination : $59-85$

ISSN : 1167-315X

Référence électronique

Alain Bernard et Sylvain Laubé, « Quelle recherche en histoire des sciences et des techniques en IUFM ? », Tréma [En ligne], 26 | 2006, mis en ligne le 29 septembre 2010, consulté le 04 mai 2019. URL : http://journals.openedition.org/trema/92 ; DOI : 10.4000/trema.92

Ce document a été généré automatiquement le 4 mai 2019.

Trema 


\title{
Quelle recherche en histoire des sciences et des techniques en IUFM?
}

\author{
Alain Bernard et Sylvain Laubé
}

1 Le travail de l'atelier 2 était consacré au thème de la recherche en histoire des sciences, comme l'indiquait le texte d'annonce des journées :

Quelles recherches en histoire des sciences et des techniques en IUFM?

- Une recherche disciplinaire universitaire

- Une recherche orientée vers la production d'outils pédagogiques

- Une recherche croisée avec d'autres champs de recherche

3 Le texte de cadrage distribué aux participants en début de première journée expliquait plus précisément le questionnement proposé :

$4 \quad$ Pourquoi les recherches en épistémologie et en histoire des sciences et des techniques (HST) ontelles leur place en IUFM? Cet atelier vise à cerner au mieux cette question, qui en cache évidemment une autre: quelles sont les recherches pertinentes en HST dans le contexte particulier qui est celui des IUFM?

5 Pour répondre à ces questions, il faut d'abord établir un état des lieux de la recherche en HST qui se fait déjà dans les IUFM. Nous examinerons à cette fin les résultats de deux enquêtes réalisées sur ce sujet pour préparer l'atelier: une enquête sur les politiques de recherche des IUFM en ce domaine, quand elles existent; une enquête adressée aux individus, chercheurs "attitrés" ou non, qui conduisent ou ont conduit des recherches en HST au sein des IUFM. Cela devrait nous permettre de définir la nature des recherches en question, sa spécificité vis-à-vis de la recherche disciplinaire classique, son degré d'institutionnalisation et de reconnaissance.

Ces constats sur l'existant montrent des richesses mais aussi des manques. Ils doivent donc permettre d'aborder la question centrale et prospective, qui est celle du type de recherche en lien avec l'HST qui devrait être promu dans les IUFM et pourquoi.

7 A partir des problèmes de fond qu'impliquent l'introduction de l'HST dans la formation des maitres et d'une perspective historique dans l'enseignement des sciences, l'atelier aura pour objet d'identifier les thématiques de recherche à faire émerger et d'examiner le cadre institutionnel 
requis au niveau national pour développer ces recherches ainsi que les collaborations avec d'autres champs de recherche que cela implique. Cela nous conduira également à poser le problème de la comparaison entre la situation française et celle d'autres pays où ces questions sont éventuellement davantage développées.

Comme indiqué ci-dessus, l'atelier a été préparé en amont par la collection de différentes informations qui ont permis d'élaborer un questionnement initial. Celui-ci a servi de fil rouge à la discussion lors de l'atelier à proprement parler.

Les parties 1 et 2 sont consacrées à une présentation de ces informations: lesquelles avons-nous tenté de réunir et de quelle façon (partie 1) ? Avec quel succès (partie 2) ? La seconde partie, consacrée aux principales circonstances du travail d'atelier à proprement parler, présente également ses principaux participants, outre les organisateurs qui sont aussi les auteurs de ce compte-rendu.

La partie 3 présente de manière détaillée les raisons qui nous ont poussé à déterminer deux grandes questions sur lesquelles nous avons orienté les débats, à savoir :

- (Q1) : Des recherches en épistémologie et histoire des sciences en tant que telles ont-elles leur place dans un IUFM?

- (Q2) : Un domaine de recherche à initier : la didactique de l'histoire des sciences ?

11 La partie 4 expose de manière synthétique les « résultats » du travail d'atelier, c'est-à-dire les éléments de réponses ou les réactions aux questionnements que nous avions élaborés. Par commodité, elle suit le découpage des deux grandes questions évoquées ci-dessus.

Enfin une conclusion récapitule le bilan qu'on peut faire de ce travail.

\section{Les données recueillies pour nourrir le travail de l'atelier}

Outre l'enquête concernant les pratiques de formation effectuée par Pierre SAVATON (voir l'article de P. SAVATON dans ce même numéro), les données que nous avions à notre disposition étaient pour l'essentiel les suivantes:

- Un questionnaire dit "institutionnel” (texte en annexe A) qui a été envoyé aux responsables de la recherche et / ou aux chargés de la communication de chaque IUFM. L'objectif était d'identifier en quoi la dimension EHST était prise en compte dans la politique de recherche des différents IUFM. Sur 32 questionnaires envoyés, 5 ont été retournés. La plus grande part de l'information a donc été recueillie en consultant la rubrique « Recherche » sur les sites Internet de chaque IUFM, dont l'information n'est pas toujours mise à jour ou reste parcellaire.

- Un questionnaire dit « souterrain » (texte en annexe B) qui a été envoyé directement (par voie électronique ou au gré de certaines réunions) à des individus travaillant à temps complet ou partiel en IUFM, qu'ils soient chercheurs « attitrés » ou non, pour cerner au mieux leur activité de recherche.

- Des informations sur le fonctionnement des IREM et plus particulièrement de la commission inter-IREM dont le thème est « Epistémologie et histoire des mathématiques ». Ces informations ont été fournies pour l'essentiel par Evelyne BARBIN (Univ. de Nantes, Centre Viète et IREM des Pays de Loire), qui est co-responsable de la commission en question. Elle est d'ailleurs intervenue brièvement à l'ouverture des journées pour exposer le travail de cette commission (voir son article dans ce même numéro). Les sites des IREM, lorsqu'ils étaient à jour, ont été consultés. 
- Des informations sur le contexte international, c'est-à-dire les groupes travaillant à un niveau international sur l'histoire et l'enseignement des sciences. Ces informations étaient très lacunaires et leur utilisation s'est bornée à la diffusion d'un texte résumant les travaux du groupe HPM (History and pedagogy of mathematics).

\section{Quelques circonstances importantes: les participants, le succès des questionnaires}

Avant d'entrer dans le détail des travaux et de ses conclusions, il convient de préciser plusieurs circonstances importantes, dont il faut tenir compte car elles induisent nécessairement un « biais » sur les discussions et leurs conclusions :

Il y avait peu de participants à l'atelier. Sur les trois proposés, c'est celui qui a eu la moins grande faveur des participants. Les participants qui ont animé l'essentiel de la discussion sont des universitaires, travaillant ou ayant travaillé en IUFM :

- Evelyne BARBIN : PU en épistémologie, histoire des sciences et des techniques, spécialisée en histoire des mathématiques, Centre Viète et IREM des Pays de Loire, Univ. de Nantes, exIUFM de Créteil

- Hélène GISPERT : PU en histoire des sciences, spécialisée en histoire de l'enseignement et des mathématiques, GHDSO de l'Univ. d'Orsay, ex-IUFM de Versailles

- Jean-Michel DUSSEAU : PU didactique de la physique, LIRDEF (IUFM de Montpellier)

- Danielle FAUQUE, docteur en histoire des sciences, représentante de la SFHST, associée au GHDSO (histoire de la chimie, de l'optique astronomique, histoire de l'enseignement des sciences), spécialiste de l'introduction de l'histoire des sciences dans le secondaire, ayant assuré de nombreux stages de formation continue en HST

- Etienne BOLMONT, IUFM de Lorraine, MCF en épistémologie (équipe EREST) et membre des Archives H. Poincaré (UMR 7117 du CNRS)

- Mario COTTRON, PU mécanique, directeur adjoint de l'IUFM de Poitou-Charentes chargé de la recherche et des relations avec l'université

- Jean-Paul ROUX, PU psychologie, IUFM Aix-Marseilles

- Alain BERNARD, IUFM de Créteil, MCF histoire et épistémologie des sciences, spécialisé en histoire des mathématiques, attaché au Centre Koyré et participant régulièrement aux travaux de l'IREM de Paris 7

- Sylvain LAUBE, IUFM de Bretagne, MCF 30e section CNU, reconverti en histoire de la physique moderne, membre du CREAD (Rennes2-IUFM de Bretagne) et attaché au Centre François Viète (Univ. Nantes)

Les «questionnaires souterrains » ont permis de réunir une information substantielle, mais extrêmement lacunaire et déséquilibrée. Vingt personnes ont répondu, qui se répartissent en 14 MCF (dont deux ayant une HDR), 1 ATER, 3 PRAG, 1 PRCE, 1 PLP ${ }^{1}$. Ce bilan révèle une série de lacunes importantes :

- Les chercheurs « attitrés » (c'est-à-dire ayant le statut d'enseignants chercheurs) sont donc surreprésentés ; de plus les six dernières personnes travaillent toutes dans un IREM. Les questionnaires ont donc pour l'essentiel manqué un de leurs objectifs, qui était d'obtenir une information sur les activités de recherche, donc nous savons pertinemment qu'elles existent mais qui ne sont pas ou très peu reconnues institutionnellement.

- Il y a par ailleurs une nette surreprésentation des historiens des mathématiques : douze sur vingt, contre cinq en Histoire Physique-Chimie et trois en Histoire SVT. Enfin, aucun 
historien des techniques n'a répondu, lacune d'autant plus importante qu'il manquait également au comité d'organisation un enseignant-chercheur dans ce domaine.

- La plus importante lacune est cependant que nous ne disposons d'aucune information concernant les formateurs travaillant dans le premier degré, alors que nous savons que plusieurs mènent des recherches isolément ou sur des projets temporaires. C'est un point très important car on sait d'une part qu'une partie importante de l'effectif des IUFM se trouve dans le premier degré, et d'autre part que l'histoire des sciences a probablement un rôle à jouer à ce niveau².

17 Les questionnaires « institutionnels » envoyés aux responsables de la recherche via la liste de diffusion trouvée sur le Portail des IUFM ont donné peu de résultats directs : seuls cinq questionnaires ont été renvoyés (un seul indiquant des recherches en lien avec l'EHST). Ce point montre la difficulté à récolter des informations. Afin de pouvoir obtenir malgré tout suffisamment de données, les informations ont été trouvées sur les sites IUFM dans la rubrique "Recherche » et leur examen permet de dresser un premier panorama. Sur trente-deux IUFM, on peut ainsi mettre en évidence des activités en lien avec l'EHST dans onze d'entre eux, comme on le verra par la suite. Ces activités sont nécessairement sous-estimées car nombre de sites ne sont pas à jour. De plus, nous manquons d'informations concernant les recherche-actions qui sont peu visibles alors qu'elles constituent une part non négligeable des recherches soutenues en IUFM.

On voit donc que toutes ces circonstances limitent nécessairement la portée des conclusions qu'on peut tirer de ces différentes sources d'idées ou d'informations. Plusieurs d'entre elles méritent d'être approfondies et complétées (comme on le verra en conclusion). Cependant, on peut raisonnablement penser que le panorama qu'elles nous ont permis de dresser dans une première approche donne les tendances les plus marquées en IUFM. De plus on voit aussi que l'absence d'information constitue dans certains cas une information en elle-même. Enfin, elles nous ont au moins permis de bâtir sur des bases à peu près objectives le questionnement qui nous a servi de fil rouge, comme on va le voir maintenant.

\section{Les deux grandes questions posées}

Rappelons les deux grandes questions qui ont orienté le travail de cet atelier :

(Q1) : Des recherches en épistémologie et histoire des sciences en tant que telles ont-elles leur place dans un IUFM?

(Q2) : Un domaine de recherche à initier : la didactique de l'histoire des sciences ?

20 Dans cette partie nous présentons tout d'abord les raisons principales qui ont conduit à choisir ces questions, ce qui nous conduira à faire une analyse plus détaillée de certaines des informations recueillies via les questionnaires, tout en tenant compte des réserves qu'il faut émettre sur leur fiabilité (cf ci-dessus partie 2).

\section{III.1. Première question. Des recherches en épistémologie et histoire des sciences en tant que telles ont-elles leur place dans un IUFM?}

21 Une des raisons du choix de cette question est son caractère a priori provocant, pour deux raisons principales. 
part, peu d'IUFM ont une politique de recherche propre, elle n'est pas absente, comme on le verra, mais sa présence reste réduite. Ceci s'explique par le fait que la recherche n'était pas ou peu encouragée jusqu'ici en IUFM puisqu'elle trouve son lieu naturel dans les universités. De ce point de vue, la situation institutionnelle de beaucoup de collègues historiens paraît en principe claire et bien définie.

- Soit ils sont enseignants-chercheurs et ils font leur enseignement à l'IUFM et leur recherche dans un laboratoire universitaire qui a passé ou non une convention avec l'IUFM.

- Soit ils sont enseignants dans le primaire ou le secondaire et formateurs à temps partagé, ou bien anciens enseignants et formateurs IUFM à temps plein, dans ce cas ils peuvent obtenir des décharges pour travailler dans un IREM (en lien avec le département ou l'UFR de mathématiques de l'université locale) ou dans un groupe de recherche-action dans certains cas. recherche, et que peu aient développé une politique très ambitieuse en général (a fortiori en histoire des sciences et des techniques), au moins un tiers des IUFM présentent en fait des activités de recherches en lien avec l'EHST et ces dernières se déclinent sous plusieurs formes :

- Des activités de recherches dans le cadre d'équipes IUFM : Caen / GRIF, Créteil / Histoire des Mathématiques (jusqu'en 2002), Montpellier / LIRDEST « Composante ERES», Nancy / équipe IUFM « EREST », Lille / « Action de recherche ».

- Des activités de recherches en lien avec d'autres types d'institutions : Aix-Marseille / ADEF (Univ. Provence-IUFM-INRP), Bretagne / CREAD (Univ. Rennes2-IUFM), Lyon / LIRDHIST (Univ. Lyon I), Versailles / INRP, La Réunion / IREM.

- Un séminaire le plus souvent en lien avec une équipe de recherche en didactique (4 IUFM : Aix-Marseille / ADEF ; Franche-Comté ; Lyon / LIRDHIST ; Bretagne / CREAD).

Une spécificité de ces recherches en lien avec un IUFM est bien évidemment que les travaux de recherches identifiés apparaissent comme tenant compte d'une dimension enseignement, celle-ci se présentant sous plusieurs aspects : didactique des disciplines, histoire de l'enseignement, analyse historique de la transposition didactique, formation professionnelle des enseignants, rôle social de la science.

28 作 clairement sous tutelle universitaire, ce qui implique en théorie qu'on ne dissocie plus la recherche et la formation, ainsi que cela se fait à l'université.

- Les enseignant-chercheurs que nous avons interrogés sont tous dans la situation d'être rattachés à un laboratoire universitaire, mais ne sont pas tous dans la situation de n'avoir aucune activité de recherche interne à l'IUFM. Six personnes sur les vingt qui ont répondu au "questionnaire souterrain » font partie d'un laboratoire de didactique bien implanté dans un IUFM, ou dépendant en partie de ce dernier, et mêlent de fait leurs recherches EHST avec des orientations didactiques. Cette donnée renvoie en partie à la question 2, à laquelle nous renvoyons donc. 
30 Pour les formateurs qui ne sont pas chercheurs "attitrés ", la comparaison entre le travail effectué en IUFM et dans les IREM est souvent éloquente : dans plus d'un IUFM, on voit une activité de recherche engageant à temps partiel des collègues enseignants à peu près nulle ou très réduite, tandis que les IREM, généralement subventionnés sur un budget universitaire ${ }^{3}$, affichent plusieurs groupes de recherche bien actifs et productifs. La comparaison donne immédiatement l'impression qu'il existe un énorme potentiel de recherche mais qu'il est sous-exploité, faute d'une institution d'accueil pour les recherches touchant à l'EHST.

- La distinction entre recherche et enseignement s'avère une fausse distinction. Elle masque en effet une spécificité de la formation des maîtres mal reconnue de fait par l'institution universitaire, à savoir son importance fondamentale pour la recherche ellemême. Cette importance est bien révélée par les questionnaires "souterrains", qui montrent, que la recherche intervient en formation de deux manières au moins (non exclusives l'une de l'autre) :

- soit un travail de recherche en EHST, élaboré par ailleurs, est réinvesti dans les formations : le cas typique est un texte ou une traduction inédite, qui fait par ailleurs l'objet d'une recherche, qui est présenté et commenté aux stagiaires,

- soit, plus profondément encore, ce sont les formations elles-mêmes qui suscitent des questions de recherche. Là encore, l'exemple type est l'édition d'un texte conçue à la fois pour la formation et pour son intérêt historique, ou bien l'organisation argumentée d'un ensemble de sources ${ }^{4}$.

- Enfin les questionnaires «souterrains" révèlent qu'une partie importante des personnes interrogées (huit sur vingt) comptent dans leur thématique de recherche des questions d'histoire de l'enseignement, qu'ils ne se poseraient pas ou moins, vraisemblablement, s'ils ne travaillaient pas dans un institut de formation à l'enseignement.

Toutes ces constatations conduisent donc à interroger sérieusement les catégories institutionnelles par lesquelles on croit pouvoir dissocier a priori la recherche en EHST et la formation des maîtres. En résumé, l'institution des IUFM montre elle-même des ambiguïtés, puisqu'elle soutient de fait, dans un nombre non négligeable de cas, des recherches dans ce domaine, tout en se refusant par principe à les accueillir, réservant cette fonction à d'autres secteurs de l'université. D'autre part, des liens "organiques " unissent de fait recherche et formation au niveau de la pratique même des membres des IUFM. Ces constatations sont évidemment liées, puisqu'une recherche conduite dans tel ou tel IUFM prend généralement son origine dans un besoin des formateurs d'enrichir leur réflexion et de conduire des actions de recherches concordantes à ce besoin.

A fortiori ces constatations conduisent à s'interroger naturellement sur ce que pourrait être le succès d'une politique de recherche plus ambitieuse dans ce domaine, c'est-à-dire à même d'exploiter le potentiel de recherche en EHST présent "de fait" sur le terrain. Le moins qu'on puisse dire est donc que la question (Q1) se pose.

D'une manière toute rhétorique, la question a été posée aux participants de leur atelier en leur suggérant de contribuer à l'argumentaire d'un éventuel « manifeste » expliquant les raisons qui font que des chercheurs en histoire et épistémologie des sciences ont leur place dans les IUFM. 


\section{III.2. Seconde question. Un domaine de recherche à initier : la didactique de l'histoire des sciences?}

Pour cette seconde question, nous sommes partis des réflexions déjà largement engagées par l'un d'entre nous (SL) au CREAD, mais dont on apercevra facilement qu'elles reflètent des préoccupations généralement partagées par d'autres équipes de recherche.

La formation des maîtres présente une dimension spécifique: elle s'occupe de la formation professionnelle d'enseignants destinés à mettre en place des apprentissages à l'Ecole primaire, au Collège ou au Lycée ${ }^{5}$. La construction des gestes professionnels repose en général sur une "épistémologie professionnelle» alimentée évidemment par les savoirs savants de la discipline enseignée (et appris à l'Université), mais aussi par des savoirs didactiques comme des savoirs épistémologiques et historiques. Dans le cas qui nous intéresse ici, le domaine concerné est celui des sciences et de la technologie. Les enquêtes effectuées par les organisateurs des journées permettent de relever plusieurs points importants :

Il existe sur un certain nombre de sites IUFM des enseignements d'Histoire des Sciences, même si ceux-ci restent malgré tout à la marge, notamment en terme d'heures de formation ${ }^{6}$.

- Les thématiques traitées ainsi que les pratiques sont très diverses et semblent dépendre des profils / compétences des formateurs responsables de ces enseignements.

- Comme on l'a vu, il existe des recherches menées dans les IUFM en lien avec l'EHST. Il semblerait que bon nombre des formateurs soient impliqués dans ces recherches ou d'autres en lien avec des laboratoires d'EHST.

A partir de ces constats, de nombreuses (et vives) questions se posent quant à la construction des gestes professionnels à l'IUFM et les formations en EHST qui existent (on se reportera à la figure I de la page suivante, qui a été utilisée lors de l'atelier pour résumer le questionnement ci-dessous).

Une première série de questions est de l'ordre de la transposition didactique :

Quels sont les savoirs "savants" de référence?

Quels sont les savoirs enseignés à l'IUFM?

Quels sont les moteurs de la transposition?

41 On peut faire une première hypothèse : les références à un savoir "savant" en EHST (ainsi que sa transposition en savoir à enseigner) sont essentiellement du domaine du formateur ou des communautés en EHST auxquelles il appartient. Il n'existe pas à l'heure actuelle de « noosphère » établie et ayant abouti à des propositions de « programmes » de formation présenté comme références et possédant des justifications concernant la formation professionnelle des maîtres. La demande institutionnelle reste donc très générale, peu contraignante, et permet des interprétations très diverses.

Une seconde série concerne les pratiques réelles et effectives de formation en EHST pour les enseignants :

Quelles sont les formes prises par la formation ? S'agit-il de conférences, d'enseignement de type universitaire (CM et TD), d'ateliers de lecture, de formation par la recherche, etc... ? Selon quelles modalités : en présentiel ou à distance?

Que sont censés apprendre les stagiaires? S'agit-il de leur faire acquérir des connaissances ou méthodologies qui sont propres à l'EHST, c'est-à-dire de "penser" I' 
EHST? Ou bien peut-on mettre en évidence une position nécessairement ancillaire de I'EHST par rapport à la didactique ? Ou bien s'agit-il de les conduire à enseigner eux-mêmes I'EHST à leurs élèves?

Comment sont évalués les apprentissages? Quels sont les obstacles à ces apprentissages? didactique :

Comment innover, inventer de nouvelles pratiques dans ce domaine et les évaluer?

Quelles sont les pistes à explorer (usage des TICE, réplication d'expériences historiques, formation par la recherche par l'étude des ressources locales, ...) ?

La question de la place et du rôle de l'EHST dans la formation des maîtres (et sans doute, par retombée, à l'Université, à l'Ecole, au Collège et au Lycée) se définira dans le futur à la lueur des éléments de réponses apportées aux questions énoncées plus haut.

Ces questions ne sont pas nouvelles en un sens, car ce sont les questions que pose en général la didactique d'une discipline donnée (celle des sciences, des techniques ou de l'histoire, par exemple). Par contre, compte-tenu du fait que toutes ces questions concernent l'enseignement de l'EHST, il s'agit clairement de poser la question nouvelle d'une didactique à initier : celle de l'enseignement de l'EHST.

Il faut ajouter, pour éviter une confusion courante, une précision importante. Les problèmes didactiques concernant l'enseignement de l'histoire ou de l'épistémologie des sciences sont en effet compris en général au niveau des enseignements scolaires euxmêmes. Or les problèmes posés ci-dessus le sont au seul niveau de la formation des enseignants, qui est la première vocation de l'IUFM et dans une certaine mesure celle des universités. Le pas est cependant très vite franchi de faire dépendre la didactique dont il est ici question de celle qui concernent directement l'apprentissage de l'EHST par le public scolaire. Des questions se posent certainement à cet égard, en particulier là où les programmes scolaires recommandent l'emploi d'un matériel à caractère "historique " (cf. les analyses proposées dans le premier atelier). On a vu plus haut, cependant, que ces questions ne sont qu'une toute petite partie des nombreuses questions qui relèvent de la didactique de l'EHST au niveau enseignant. Réduire le tout à la partie fait donc perdre à cette dernière l'essentiel de son intérêt. En termes plus simples, l'intérêt essentiel d'enseigner l'EHST à des enseignants ne se réduit nullement à leur faire répéter le même enseignement pour leurs élèves ; au contraire, l'intérêt pour eux d'une formation en EHST dépasse de loin cette question particulière et a des implications plus vastes sur la qualité de leur enseignement. 


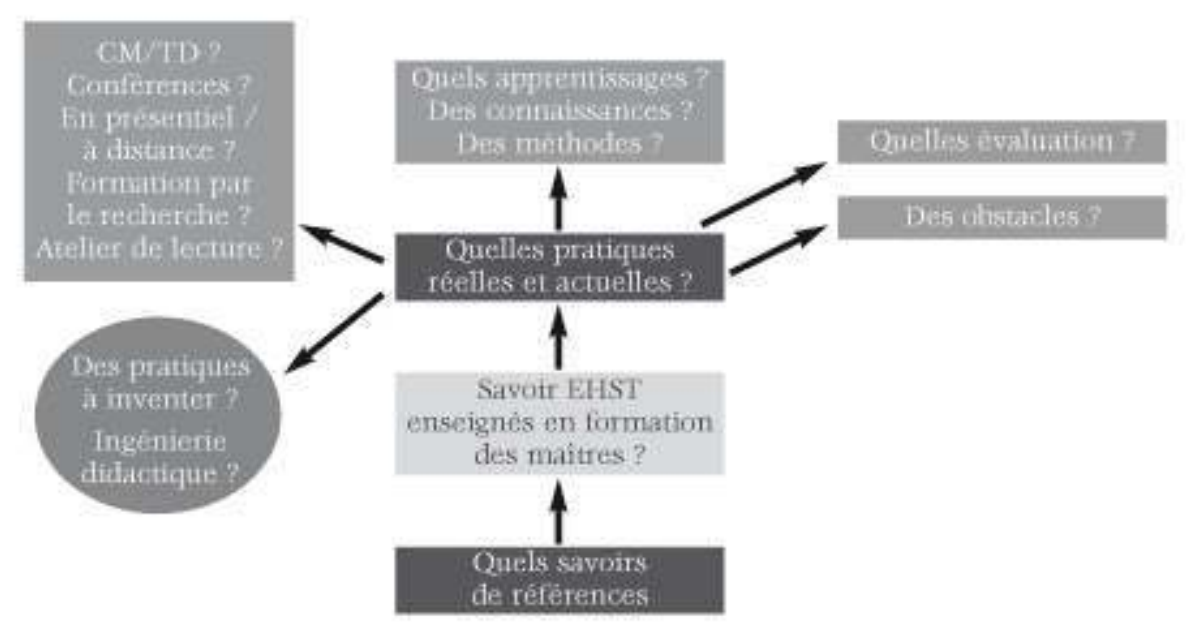

Fig. I Les questions générées par l'enseignement de l'EHST dans les IUFM.

\section{La synthèse du travail en atelier}

Les réactions aux deux questions présentées ci-dessus ne sont pas vraiment comparables. Quitte à simplifier, on peut dire grosso modo que les réactions à la première question ont été à chaque fois relativement consensuelles et ont donc servi de "boîte à idées", notamment dans la perspective du «manifeste dont il a été question plus haut (cf III.2., fin). La seconde question, par contre, a reçu un accueil très différent et a permis de mettre en évidence des désaccords profonds sur la recevabilité de l'idée sous-jacente : les uns comme on le verra, ont rejeté d'emblée cette idée comme prématurée, les autres l'ont au contraire acceptée immédiatement comme étant naturelle.

Cette présentation, comme on le verra, est un peu simplifiée, dans la mesure où la discussion sur la première question a fait aussi apparaître certaines tensions révélatrices, et où une discussion a eu lieu sur la seconde qui a conduit notamment les participants qui refusaient le plus l'idée initiale à nuancer leur point de vue. Néanmoins, cette diversité de réactions explique que notre synthèse n'a pas la même forme dans les deux cas : pour la première question, elle revient pour l'essentiel à faire une liste, que nous espérons la plus exhaustive possible, des nombreuses et riches idées qui ont été proposées pour y répondre de manière convaincante; pour la seconde, elle revient plutôt à faire pour l'essentiel l'inventaire de différents types de réactions à une proposition de cette nature.

Dans les deux cas, il apparaîtra clairement que les échanges ont été très riches, même quand (ou peut-être surtout lorsque) ils étaient conflictuels et ils attestent de la qualité des débats. Nous espérons avoir restitué ici la richesse de ces échanges, même s'il n'a pas toujours été possible de restituer tous leurs détails. Pour ne pas allonger le texte nous avons abrégé la plupart du temps les nom et prénom des participants par leurs initiales (JMD = Jean-Michel Dusseau, HG = Hélène Gispert, etc.) 


\section{IV.1. La première question}

50 On peut résumer les débats qui ont eu lieu en trois grands points, qui suivent grosso modo un ordre de « délicatesse croissante »:

1. Les questions de conservation du patrimoine scientifique et / ou scolaire, liées éventuellement à des problématiques d'histoire de l'enseignement éventuellement croisées à des problématiques didactiques, sont apparues dès le départ comme une problématique relevant de l'EHST et spécifique aux historiens travaillant en IUFM.

2. Concernant toutefois la place en IUFM des historiens et épistémologues des sciences qui ne travaillent pas sur une "problématique spécifique" (aux IUFM), les constatations faites sur le travail qu'ils y font de fait actuellement conduit à souligner la cohérence de ce travail avec un objectif universitaire : la formation par la recherche; et avec un objectif des politiques de recherche en IUFM : le réinvestissement des recherches en formation. Ce débat était évidemment fortement lié aux questions relatives à l'intégration (promise) des IUFM dans les universités et a soulevé à son tour des interrogations importantes sur l'avenir.

3. Sous un troisième point de vue enfin, on s'est interrogé sur les priorités des politiques de formation et de recherche des IUFM, et plus particulièrement sur le fait que la recherche de formations "directement utiles" aux enseignants sur le terrain conduisait naturellement à marginaliser l'apport des historiens, considéré comme d'intérêt secondaire et "culturel". Quelles réponses faut-il alors apporter au questionnement qui paraît a priori légitime des responsables de formation en IUFM?

51 Nous reprenons chacun de ces points plus en détail ci-dessous.

\section{1 / Les problématiques de recherche en EHST spécifiques aux IUFM}

52 Cette question a été débattue dès le début du travail en atelier, puis plus tard à la suite d'une discussion initiée lors de la pause de la séance du vendredi. Le débat initial s'est basé sur l'exemple concret fourni par Jean-Michel DUSSEAU, qui a rapidement résumé le travail envisagé à Montpellier d'exploration d'un fond documentaire de manuels scolaires anciens, sur lequel un double regard, de didacticien et d'historien, lui paraît non seulement souhaitable mais nécessaire. Pour illustrer ce "croisement des regards" sur un même matériel, il a pris l'exemple de son propre travail sur l'introduction du concept d'électron dans l'enseignement secondaire, qu'on peut suivre au fil des diverses éditions de manuels en première. Il $\mathrm{y}$ voit donc un exemple paradigmatique de situation où la présence d'un historien en IUFM ${ }^{7}$ est justifiée et permet un regard croisé d'historiens et de didacticiens sur le même objet (ici un corpus scolaire).

Sur la base de cette présentation "consensuelle" des rapports possibles entre approches didactique et historique, une discussion s'engage à partir des réticences exprimées par Hélène GISPERT, elle-même historienne de l'enseignement. Elle pense plus précisément qu'une approche didactique de ce type de matériel historique peut conduire à des biais inacceptables pour un historien, et elle insiste donc pour qu'on fasse bien la différence entre les deux démarches. Elle cite par ailleurs l'exemple des recherches encouragées à l'IUFM de Versailles, développées en particulier par cinq chercheurs qui sont aujourd'hui PRAG à l'INRP et poursuivent des recherches sur l'histoire de l'enseignement, en dehors de toute problématique didactique. Malgré cela, ces recherches sont liées à une formation de formateurs en PE et PLC2. Il s'agit donc d'un exemple d'une double activité de recherche et de formation qui n'est pas orientée par les questions didactiques. 

"non attitrés" soient poussés, parce qu'ils ont une visée de formation d'enseignants, à examiner (et, dans certains cas, exhumer) des collections de manuels ou d'artefacts qui n'intéressent pas forcément (ou pas autant) d'autres universitaires. Le débat de Montpellier permet pour l'instant de dégager au moins trois niveaux d'investigation de ce genre de question :

- Un niveau où il s'agit, de la part d'historiens spécialistes de ce genre de travail, d'exhumer des archives d'instruments ou de manuels et d'en faire un inventaire.

- Un niveau de critique historique à proprement parler de cet inventaire (comparaison avec d'autres archives, inscription dans un histoire longue de l'enseignement et des sciences).

- Un niveau de critique didactique, où il s'agit d'exploiter cet inventaire pour nourrir une réflexion didactique sur l'évolution et la nature des contenus d'enseignement.

\section{d'investigation:}

Faut-il laisser ces trois niveaux se croiser (c'est le point de vue semble-t-il de JMD pour les deux derniers au moins) ou les maintenir suffisamment distincts (HG)?

Etant entendu que bien souvent la motivation de ce genre de recherche part d'un problème de formation (exhumer un «matériel » ré exploitable pour la formation d'enseignants, c'està-dire pour nourrir la réflexion de ces derniers et enrichir leur pratique pédagogique), quel lien faut-il faire «en aval », c'est-à-dire entre les résultats de ce genre de recherche et les formations qu'on peut développer sur leur base?

58

Il convient enfin de remarquer que les deux derniers « niveaux d'investigation » ne sont pas forcément liés au premier, au sens qu'une réflexion sur l'histoire de l'enseignement ou de ses artefacts peut très bien se développer sur un matériel déjà publié ou mis à jour, mais qu'il s'agit par exemple d'examiner sous un angle nouveau. Dans tous les cas, un inventaire plus complet de ces activités et des échanges auxquels ils pourraient ou devraient donner lieu est à poursuivre.

Tréma, 26 | 2006 


\section{2 / La place des recherches en EHST “non spécifiques" en IUFM} les questions ici débattues ne peuvent pas se limiter à l'identification de problématiques de recherche en EHST spécifiques aux chercheurs, attitrés ou non, qui travaillent en IUFM. Un certain nombre de ces derniers, en fait leur majorité, ont une activité de recherche qui ne se distingue pas fondamentalement de celle d'un universitaire travaillant dans un autre contexte mais dans le même domaine, celui de l'EHST. Faut-il alors considérer que la place de cette activité (ou plutôt de cette partie de leur activité) est précisément à l'université, et ne concerne que de manière très secondaire les IUFM ?

contrer cette idée trop simple, pour ne pas dire franchement fausse, on rappelle les arguments principaux qui infirment cette idée (cf. p. 63 III. 1.) et qui ont été rediscutés au cours de l'atelier :

- a) L'expérience des IREM (pour les mathématiques) et des groupes de recherche-actions existant dans différents IUFM montre le très grand 'potentiel de recherche' existant dans ce domaine en IUFM. Il est bien ici question de véritables recherches en EHST, dont la seule particularité est de trouver leur lieu dans un type d'institution adapté à leurs acteurs, c'està-dire des enseignants.

- b) et c) Les questionnaires permettent de constater une certaine osmose entre recherche et formation, pour deux raisons principales :

- b) La recherche conduite en EHST par des formateurs est très souvent utilisée ou réutilisée en formation, et dans certains cas la formation sert de "terrain d'essai“ à un premier exposé de travaux de recherche.

- c) Surtout, la formation, c'est-à-dire l'activité de formation conduit à développer de nouvelles problématiques de recherche en EHST, et cette réflexion a non seulement lieu devant des enseignants, elle les associe de fait à cette élaboration. C'est même précisément cette « association » qui permet assez souvent d'adjoindre des enseignants qui participent à une formation à des groupes de recherche plus poussée. La formation sert alors de « lieu de recrutement » pour ces derniers.

61 Ces deux derniers points montrent que l'activité de formation est toujours, en partie au moins, une activité de recherche, soit parce qu'elle est lui est liée (b), soit parce qu'elle est de fait une activité de recherche ou bien son point de départ (c).

Sur ces trois points la discussion a permis d'apporter les éléments suivants.

63 objectif explicite des politiques de recherche en IUFM, lorsqu'elles existent: le réinvestissement de recherches en formation. En réalité, MC argumente que la plupart des MCF ne réinvestissent pas leurs recherches dans la formation, à part au niveau universitaire (en Master). Si les collègues historiens réussissent à le faire, ils répondent de facto aux ambitions signalées ci-dessus. Cela signifie aussi qu'ils ont tout intérêt à valoriser cette dimension de leur enseignement pour la faire reconnaitre par l'institution. Ce point important sera repris dans la discussion de l'aspect (3 / ).

Sur le troisième point (c), plusieurs participants, à commencer par EB, font remarquer qu'il correspond à un objectif que la politique universitaire de formation valorise de plus en plus depuis les dernières années: la formation par la recherche. L'idée est que la formation universitaire, qui est spécifiquement liée à une activité de recherche, puisse être considérée comme une formation professionnelle. C'est ce à quoi visent, dans les 
universités, des manifestations telles que les doctoriales, où les étudiants sont invités à valoriser leur formation

universitaire pour préparer leur « insertion professionnelle ». En IUFM, cette exigence se retrouve en certains endroits, comme l'écriture de mémoires professionnels pour les stagiaires en formation initiale. L'enjeu latent de ce débat est bien sûr l'harmonisation de la formation dispensée en IUFM avec les cursus universitaires modernes et notamment la validation du diplôme de Master.

Le premier point (a) est progressivement abordé dans la suite de la discussion et d'une manière qui mérite d'être relatée en détail : $\mathrm{EB}$, poursuivant le raisonnement précédent, fait remarquer que puisque le destin des IUFM est d'être intégrés à l'université (ou davantage qu'ils ne le sont aujourd'hui), la question soulevée, à la limite, ne se pose pas. Plus exactement selon elles, ce sont d'autres questions qui se posent :

- Quelles sont les recherches en EHST et en lien à l'enseignement possibles à l'université, quand elles ne relèvent pas de la didactique des sciences? C'est ici qu'elle cite le cas, évoqué plus haut, de la collègue enseignante. EB cite, par opposition, le cas de la Belgique, où des thèses de ce type (c'est-à-dire historiques, tout en étant liées à des questions de formation) sont possibles. Ce point est important car c'est la seule fois dans tout l'atelier où la comparaison entre les situations française et internationale a été faite. Mais cet exemple unique fait deviner les enseignements qu'on pourrait en tirer.

- D'autre part, gagnera-t-on à faire entrer les recherches conduites en IUFM sous le régime universitaire tel qu'il se dessine actuellement, impliquant une mise en concurrence des laboratoires et une concentration de ces derniers en quelques "pôles", généralement parisiens. Ne risque-t-on pas de perdre une spécificité des IUFM, semblable à celle des IREM, qui est leur caractère "provincial" et décentralisé ?

Dans tous les cas cependant, la question semble de fait résolue de fait par le passage obligé d'une formation détachée de la recherche à une formation par la recherche, au même titre que d'autres formations universitaires. Cette évolution préfigurée est résumée par un lapsus amusant d'EB, qui parle à un moment donné de l'intégration « des IUFM dans les IREM » (au lieu des universités).

Un débat s'engage cependant à ce sujet, car d'autres participants soulignent que cette évolution est loin d'avoir un caractère évident, pour la simple raison que l'enjeu essentiel de cette intégration est le destin de la formation des professeurs des écoles, qui reste de fait (et de loin) la mission "quantitativement" la plus importante des IUFM ${ }^{11}$. Or on peut s'interroger sur ce que sera le devenir de la formation des professeurs des écoles dans une hypothétique mise sous tutelle universitaire des IUFM. Quel département universitaire est-il apte à accueillir une formation qui ne se rattache à aucun département disciplinaire en particulier? S'il est imaginable, pour la formation dans le second degré, que chaque département de sciences prenne en charge la formation des professeurs relevant de la discipline concernée - c'est ce que le lapsus d'EB préfigure pour les mathématiques. Mais qu'en sera-t-il pour le primaire? Ne doit-on pas redouter que le passage dans un département ad hoc ne reproduise au sein de l'université le cloisonnement entre formation et recherche qu'il s'agissait précisément d'éviter?

Cet enjeu global rejoint une des grandes lacunes des informations recueillies pour alimenter le travail de l'atelier : la formation dans le primaire est en effet de manière évidente sous-représentée dans les débats. Ce point mérite réflexion, car cette absence de représentation ne correspond pas à la réalité de l'enseignement et de la formation au niveau primaire, où l'HST est nettement présente. Autrement dit, le 'potentiel de 
recherche' auquel il était fait allusion plus haut est bien présent à ce niveau aussi, mais nous n'en avons pas pour l'instant de représentation claire. Ce déséquilibre mérite qu'on réfléchisse aux moyens de pouvoir refléter à l'avenir cette activité et l'incorporer à nos réflexions.

\section{3 / La question de l'ordre de priorité donné aux recherches en EHST parmi les objectifs de formation et de recherche des IUFM}

Le débat prend un nouveau tour alors que JMD reprend une remarque très pertinente faite le matin même par Jean-Jacques Dupin lors de la table ronde introductive. La question soulevée alors est celle de la place de l'EHST dans les priorités qui sont traditionnellement celles d'un IUFM. Si en effet on résume brutalement ce qu'est la préoccupation traditionnelle et première des responsables de formation et de recherche en IUFM, alors celle-ci n'est pas de promouvoir des structures ou des formations qui ne permettraient jamais (aux yeux de ces responsables) que d'enrichir la culture personnelle des enseignants (quel que soit l'intérêt qu'on reconnaît par ailleurs à ces démarches), mais bien de résoudre les problèmes considérés comme "plus urgents" que doivent affronter les enseignants au quotidien. Si on ajoute à cela le paramètre essentiel que constituent des budgets de formation généralement serrés, il faut s'attendre à ce que ce soit vers d'autres types de recherche et de formation que se tournent les responsables d'un IUFM. Comment des historiens, dans ce contexte, peuvent-ils justifier leur existence et leur travail ?

De ce point de vue, le débat permet d'explorer plusieurs réponses à cette question, probablement la plus « épineuse » des trois problématiques examinées dans cette partie.

Une première réponse est développée de nouveau par JMD, qui défend depuis le début une association intelligente entre une démarche didactique et une démarche historique et / ou épistémologique. Traditionnellement, rappelons que les rapports entre les deux peuvent être résumés à une vision "ancillaire ", la didactique étant la voie d'accès prioritaire aux questions posées par l'enseignement et se subordonnant au titre «d'outils» ou de «servantes" (parmi d'autres) à l'histoire ou l'épistémologie des sciences. Cette vision soulève des problèmes spécifiques qui ont été discutés de manière plus approfondie dans le traitement de la seconde question (Q2). Ici, JMD défend une vision plus subtile et qu'on peut résumer en deux points principaux :

(a) tout d'abord il souligne que la didactique des sciences n'est pas moins que l'EHST victime de la vision "utilitariste» de la formation et de la recherche qu'on a résumée plus haut. Restreindre la nécessité des formations à celle de résoudre des "problèmes urgents » revient à occulter une dimension essentielle du travail du didacticien, que JMD résume en disant qu'il se sent « une responsabilité pour les contenus » (disciplinaires);

(b) c'est à ce titre, continue-t-il, qu'il est naturel que les didacticiens collaborent avec des historiens pour défendre cette réflexion sur le long terme sur les contenus disciplinaires, leur valeur et leur évolution. Ici encore c'est la philosophie adoptée à l'IUFM de Montpellier qu'il défend.

Il reste évidemment à déterminer, comme on l'a souligné plus haut, si cette vision est locale à Montpellier ou bien est partagée par d'autres praticiens et théoriciens de la didactique. La situation est probablement loin d'être simple, comme le révèle bien notre enquête institutionnelle, et mériterait d'être approfondie. 

qu'on peut justifier la présence d'historiens en IUFM comme "collaborateurs" de didacticiens, quelle que soit la façon dont cette collaboration soit par ailleurs conçue; étant entendu qu'on peut également chercher à les «justifier » par les exigences d'un enseignement historique pour les élèves; existe-t-il au delà de ces arguments de vraies raisons de poids pour défendre la présence en IUFM de chercheurs en histoire? A quoi peuvent-ils contribuer en tant que ce qu'ils sont en propre, c'est-à-dire des historiens et épistémologues?

81 Cette question avait servi de conclusion au travail du vendredi, mais elle n'a été véritablement développée que lors dans le travail du samedi et grâce à l'intervention répétée d'HG. On peut résumer le "remue-méninges » sur ce point en disant que la question revient à défendre la place des historiens en IUFM en l'articulant de manière claire à plusieurs des objectifs explicites que s'assignent traditionnellement les IUFM. Au 
fil de la discussion, HG propose finalement de présenter les choses sous la forme de quelques questions simples, qui toutes renvoient aux objectifs en question :

- Entend-on engager ou non les enseignants à une réflexion sur la place qu'occupe leur champ disciplinaire, leur institution (l'école), leur métier dans la société ? Si la réponse est oui, alors un IUFM a besoin d'historiens parmi les experts de ces questions, car il n'y a pas de société qui n'ait d'histoire. En outre il n'est plus à l'heure actuelle d'historien des sciences et techniques qui ne tienne compte du contexte social et culturel de cette histoire, ce qui les rend d'autant plus compétents pour aborder ces questions.

- Entend-on donner aux enseignants une idée précise des contenus des programmes disciplinaires qu'ils ont à enseigner, non pas en tant que "tables de la loi" à respecter à la lettre, mais en tant que produits complexes d'une évolution historique qui explique à la fois leur forme et leur rapport avec les programmes d'autres disciplines? Veut-on on non leur donner ainsi une compréhension plus fine du "pourquoi" de ces programmes? Si la réponse est oui, alors l'IUFM a de nouveau naturellement besoin des experts de cette question que sont les historiens de l'enseignement des sciences.

- Entend-on proposer aux enseignants une réflexion riche sur les contenus disciplinaires qu'ils ont à enseigner ainsi que leurs rapports aux différentes sciences et techniques dont ils relèvent en partie ? Si la réponse est oui, alors les IUFM doivent convoquer, parmi les experts susceptibles d'alimenter cette réflexion, des historiens, car il n'y a pas de science qui n'ait d'histoire.

Plusieurs des points évoqués dans cette série de questions croisent des thèmes évoqués tout au long du travail. Ce qui leur est original, cependant, est moins le contenu que la façon de les articuler de manière claire et explicite à plusieurs des objectifs traditionnels des IUFM, dont on peut penser qu'ils ne seront nullement rendus caducs par le passage sous tutelle universitaire. Ce travail d'explicitation est évidemment indissociable d'un travail de valorisation des activités existantes dans les quelques groupes de recherches travaillant sur ces questions - ce sur quoi nous reviendrons en conclusion.

\section{IV.2. La seconde question}

83 Cette question d'initier des recherches en didactique de l'EHST s'est trouvée être étonnante, voire provocante, car les réactions ont été vives et le débat riche: les uns, comme on le verra, ont rejeté d'emblée cette idée (au moins) comme prématurée, les autres l'ont au contraire acceptée immédiatement comme étant naturelle.

Les positions complètent celles observées lors des débats sur la première question, en particulier celles développées par HG, EB et JMD. On retrouvera en particulier chez HG et EB la nécessité de bien séparer les « territoires disciplinaires », les méthodologies propres de l'EHST et de la didactique.

85 L'analyse de l'enregistrement des débats permet de dégager deux points de vues contradictoires. Cependant si cette contradiction s'est révélée forte, le discours s'est trouvé plus nuancé en fin d'atelier. Plutôt qu'une transcription des débats, nous avons donc cherché à mettre en évidence les types de points de vue.

Dans le premier, HG et EB se retrouvent pour déclarer sinon leur opposition, du moins leur gêne.

87 - Pour HG, la question est étrangère à l'HST, voire dangereuse par un risque de perte d'identité face aux sciences de l'éducation. Ses interrogations spontanées sont 
nombreuses: quelles armes, quels outils, quelle formation sont elles nécessaires pour traiter ce problème? Qui peut les mener, dans la mesure où les historiens des sciences ne sont pas les mieux armés pour de telles questions? Une recherche qui dépendrait de quelle matrice générale? Par la suite, elle concède qu'il s'agit sans doute d'une bonne question / d'une question réelle mais juge a priori que la communauté des historiens des sciences et des techniques n'est pas assez mûre et ne possède pas les moyens pour la traiter sérieusement : on ne trouvera pas d'historien prêt à s'investir dans ce domaine et capable de donner une recherche de qualité. Ce point de vue fut réfuté à partir des résultats des deux enquêtes : il existe des chercheurs en EHST à l'IUFM possédant des compétences en didactique, participant à des recherches en didactique et susceptibles de constituer un vivier pour développer ce domaines. Pour HG, cette question reste à la marge et pose des problèmes de recevabilité au sein de la communauté EHST : afficher une telle question de recherche dans l'éventualité d'un manifeste peut rendre trouble ou flou un positionnement des recherches en EHST possibles au sein des IUFM. Pour HG, il n'est pas souhaitable de se retrouver dans la position de mépris où se trouve la didactique aux yeux de nombreux mathématiciens. Nous reviendrons plus loin sur cette réaction, qui nous semble très caractéristique du débat sur ces questions parmi les mathématiciens français.

A partir de l'évocation de ses relations avec les didacticiens des mathématiques avec lesquels elle a travaillé sur des questions communes, EB indique des difficultés: les concepts de la didactique sont étrangers à l'HST qui n'a pas à leur servir de caution. Elle cite deux exemples : des recherches effectuées en collaboration avec des didacticiens dont les conclusions étaient traduites de la part de ces derniers comme une validation des concepts de théories didactiques et une opposition avec Y. CHEVALLARD sur la question d'aborder l'histoire des sciences avec les mains pleines (de concepts didactiques) ou "à mains nues" (en référence à P. Veyne). EB conteste par ailleurs la scientificité du didactique et invite à faire attention aux aspects prescriptifs, aux normes qui peuvent être induites par les recherches pédagogiques et qui ne lui semblent pas souhaitables. Pour EB, il existe bien des problèmes dans l'enseignement de l'EHST, comme la lecture contextuelle des textes anciens, où la didactique n'apportera pas de réponses. EB propose de remplacer le mot didactique par formation, en indiquant qu'elle entend, par là, formation par la recherche (basée sur la littérature primaire) ce qui justifie la présence d'enseignants-chercheurs. Elle termine en indiquant que le problème est en fait complexe, car les recherches didactiques sont diverses et des collaborations fructueuses entre didacticiens et historiens existent (IREM de outre qu'il s'agit bien d'un domaine à inventer: la didactique de l'HST et que cela poserait sans doute des problèmes de reconnaissance au sein de la communauté HST.

Les autres participants indiquent qu'il existe des obstacles mais que l'idée n'est pas incongrue, qu'on en est qu'aux balbutiements compte tenu de l'inexistence d'une communauté de recherche sur ce sujet. Enfin, à partir du constat qu'on peut se réjouir du développement important de sites Internet ${ }^{12}$ où on peut trouver de la littérature primaire de très bonne qualité, mais accessible essentiellement à des experts, la question de l'élaboration d'outils informatiques adaptés aux enseignants (ou plus largement) aux étudiants amène à montrer qu'au-delà des points de vues contradictoires il $\mathrm{y}$ a des préoccupations très Lyon par exemple). EB doit malheureusement quitter la séance à ce moment-là.

Le second point de vue est clairement favorable. 
91 En contre-point d'HG, JMD indique qu'historiquement ce sont des mathématiciens et des physiciens qui ont monté la didactique des sciences : son cas personnel en est un exemple. Issu de la 28e section, il est resté en $28 \mathrm{e}$ en tant que didacticien de la physique et il se définit comme épistémologue appliquée à l'enseignement. Sur ce modèle, seuls des historiens des sciences pourront développer ce type de recherches. Il précise en proches de part et d'autre, ce qui augure des possibilités de travailler en commun sur ce type de thématiques.

92 En ce qui concerne la seconde question, on peut sans doute résumer l'atelier de façon un peu lapidaire par la proposition suivante : la question de l'enseignement de l'EHST est une question «socialement urgente» (si l'on reprend les mots d'HG). Son niveau de traitement (c'est-à-dire sa traduction en termes de recherche par l'identification de questions et de méthodes de recherche spécifiques) va dépendre de la capacité d'une communauté de chercheurs capables de s'en emparer et de produire une recherche de qualité. A l'heure actuelle, se pose la question de savoir si la communauté liée à l'EHST est mûre ou non. Il est clair que la nouveauté de la question nécessite un effort d'explicitation sur ce qu'il y derrière les mots «didactique de l'EHST en formation des maîtres ».

93 Par ailleurs, la question de la recevabilité de cette question au sein de la communauté EHST ayant été évoquée, on peut dire qu'elle est liée à des positions très contrastées par rapport au mot didactique (et aux didacticiens). Ce point indique bien que le discours tenu par chacun des participants est sous-tendu par sa propre représentation de la didactique. La didactique des mathématiques soulève des réactions vives qui sont les marques d'une controverse existante. Or cette situation conflictuelle semble propre, aux mathématiciens d'une part, aux Français de l'autre. Ces questions ne semblent pas soulever les mêmes émotions dans le cas par exemple de la physique, comme en témoigne au moins le point de vue présenté par JMD, ni exister au même degré dans des pays étrangers (Canada, Belgique ...).

Enfin si les points de vue sont contrastés, il est intéressant de noter que la partie Q1 de l'atelier permet d'élaborer des éléments de réponses à des questions qu'on peut considérer comme relevant du domaine de la didactique de l'EHST en formation des maîtres (voir figure p. 69). Par exemple, une proposition de modalité professionalisante au sein de la formation IUFM: la formation par la recherche et trois objectifs de formation possibles ont pu être signalés : 1) réfléchir sur la place qu'occupent les champ disciplinaires, l'institution (l'école), le métier d'enseignant dans la société, 2) comprendre et connaître les contenus des programmes disciplinaires en tant que produits complexes d'une évolution historique, 3) réfléchir sur les contenus disciplinaires à enseigner ainsi que leurs rapports aux différentes sciences et techniques dont ils relèvent en partie.

\section{Conclusion}

Cette conclusion sera lapidaire sachant que les points les plus saillants seront repris et mis en regard des autres résultats des journées de Montpellier dans la synthèse (qu'on trouvera en fin de numéro).

\section{1 / Les acquis positifs sur les deux grandes questions travaillées}

- Des problématiques EHST spécifiques aux IUFM semblent exister. 
- On peut montrer l'existence de liens entre formation et recherche en EHST. Il reste à valoriser cette richesse au niveau des institutions universitaires.

- Un lien "riche" entre recherches historiques et didactiques peut être développé.

- On peut défendre la fonction des historiens en IUFM de manière directe, en articulant l'argumentaire sur les objectifs classiques des IUFM (citoyenneté, réflexion sur les programmes d'ensgt, réflexion sur les contenus scientifiques).

\section{2 / Les questions pendantes, celles qui ont été posées ou non, sans réponse dans les deux cas (et qui nécessiteraient un complément d'enquête ou information)}

- L'idée d'une didactique des sciences semble d'emblée acceptable par des didacticiens de la physique ; acceptable mais peu réaliste par les historiens. Pourquoi ?

- Quelle part des chercheurs IUFM est concernée par des questions de recherche sur la conservation du patrimoine (manuels + artefacts)?

- Comment se déclinent les questions de recherche en lien avec le primaire?

- Comment toucher les "chercheurs non attitrés" et les associer à aux réflexions initiées ici?

- Comment valoriser le travail de recherche EHST qui a lieu au sein de la formation ou qui se fait pour la formation?

\section{3 / Un point important à développer afin de mieux décrire l'état actuel des lieux en terme de recherche et développer des axes} futurs.

La question des activités de recherches en lien avec la formation des maîtres dans le cadre international. En effet, ce point est fondamental si l'on souhaite entrer dans une dynamique riche s'appuyant aussi sur l'expérience et les pratiques de nos collègues étrangers.

\section{ANNEXES}

\section{Annexe 1}

\section{Texte du questionnaire « institutionnel »}

«HST et recherches dans les IUFM »

IUFM :

Responsable de la Recherche :

Coordonnées : 


\section{La politique de recherche de votre IUFM présente-elle une composante en lien avec l'Histoire des Sciences et des Techniques?}

圈 oui 圈 non

\section{A) Si oui, sous quelles formes?}

- a) Une recherche en lien avec d'autres domaines de recherche (par exemple : didactique des sciences)?

咠 oui 㜔 non

- b) En collaboration avec une équipe de recherche universitaire (ou un autre type de partenaire)?

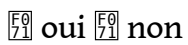

Si oui

Nom du laboratoire (ou du partenaire) :

Nom du responsable de la recherche :

Nombre de chercheurs impliqués :

Merci d'en préciser le(s) sujet(s) :

- c) Dans le cadre d'une recherche-action?

㜔 oui 疄 non

Si oui

Nom du responsable :

Nombre de chercheurs / formateurs impliqués :

Merci d'en préciser le(s) sujet(s) :

- d) Dans le cadre d'un séminaire de recherche (ou d'un colloque)?

Si oui

Nom du responsable :

Thématique :

Fréquence :

B) S'il n'existe pas de recherche en lien avec l'Histoire des Sciences et des Techniques

Pourriez-vous indiquer les éventuelles raisons que vous pourriez identifier (par exemple : absence de ressources humaines? etc.)

C) Remarques et informations complémentaires : 
Questionnaire à renvoyer par mail ou courrier à :

S. Laubé, IUFM de Bretagne.

CREAD / Site de Brest, 8 rue d'Avranches 29200 Brest

Tél. 0298478038

\section{Annexe 2}

\section{Texte du questionnaire « souterrain »}

\section{JOURNEES D’ETUDE HISTOIRE DES SCIENCES : FORMATIONS ET RECHERCHES EN IUFM}

IUFM de Montpellier, les 20 et 21 mai 2005.

QUESTIONNAIRE INDIVIDUEL SUR LES RECHERCHES HST EN IUFM.

Tu trouveras ci-dessous un questionnaire sur tes activités de recherche au sein et en dehors de l'IUFM qui ont un lien avec l'histoire des sciences et des techniques (HST).

La finalité générale de ce questionnaire est de faire un état des lieux aussi précis que possible de la place qu'occupe l'HST et les problèmes didactiques qui y sont liés dans les politiques de recherche des IUFM ; plus particulièrement, il s'agit ici de cerner les difficultés que des collègues ont pu rencontrer (ou non) pour développer isolément des projets de recherche dans ce domaine.

Il est adressé à toute personne qui est formateur ou formatrice à temps plein dans un IUFM, ou bien qui y intervient ponctuellement (formateurs associés des IREM notamment).

Un autre questionnaire vise à définir comment ont été définies les orientations de la politique de recherche dans de domaine ; tu peux aussi le renseigner si tu as des informations à ce sujet que nous n'avons pas pu recueillir (voir posters et questionnaire " politique de recherche »).

Nous te remercions de prendre le temps de répondre à ces quelques questions. Il est possible de communiquer aux organisateurs tout document explicitant un point ou un autre, par exemple la présentation d'un groupe de recherche ou d'une publication (passés ou en cours).

Les résultats des deux questionnaires feront l'objet d'une synthèse qui sera publiée en ligne et dans la revue Tréma prochainement.

Les organisateurs.

- 1 L'IUFM où tu interviens :

- 2 Tu es : MCF / PR / habilité à diriger des recherches / PRAG / PRCE / PLP / autre (ingénieur de recherche..).

- 3 Quelles sont tes principales thématiques de recherche qui ont un lien avec l'HST ?

- 4 Participes-tu ou animes-tu un groupe de recherche à l'IUFM en lien à l'HST ? Lequel ? (détailler éventuellement).

- 5 Par ailleurs, exerces-tu tes recherches en lien avec l'HST dans un laboratoire ou un autre institut? Si oui :

a) Lequel ? 
b) Dépend-il de l'IUFM ? Est-il associé à l'IUFM par une convention, un contrat, ou autre?

- 6 Les recherches que tu fais ou a faites en lien avec l'HST ont-elles donné lieu à des publications? Si oui :

a) Lesquelles ? (indiquer éventuellement un site où elles sont référencées).

b) Les recherches avaient-elles pour but cette publication?

- 7 Les formations que tu fais en lien avec l'HST à l'IUFM sont-elles liées aux recherches que tu fais ou a faites? De quelle manière?

- 8 Si tu es enseignant-chercheur :

a) Quelle est ta section CNU ?

b) Lors de ton recrutement à l'IUFM, t'a-t-on demandé de développer tes recherches en relation à l'HST, ou au contraire dans un autre domaine ? (Préciser).

- 8bis Si tu es professeur (Agr ou Cer) :

a) As-tu reçu une formation en HST ? si oui laquelle?

b) As-tu soutenu, ou prépares tu une thèse?

c) As-tu candidaté, et / ou vas tu candidater, sur un poste d'enseignant-chercheur?

- 9 Les recherches ayant rapport à l'HST que tu mènes éventuellement au sein de l'IUFM :

a) Sont-elles soutenues financièrement par ce dernier?

b) Sont-elles soutenues par un financement extérieur type ACI ou autre?

c) Font-elles l'objet d'une collaboration avec d'autres domaines (didactique, histoire de l'éducation, sciences de l'éducation, sociologie, etc.) ? (détailler).

d) Sont-elles à ton avis globalement soutenues par l'IUFM, ou le contraire? (détailler).

- 10 As-tu par le passé essayé, sans succès, de promouvoir ou d'organiser dans ton IUFM des recherches en lien avec l'HST et / ou sa didactique? Si oui :

a) Lesquelles ? (détailler).

b) Pourquoi la tentative n'a-t-elle pas abouti?

- 11 Autres remarques, suggestions, ou précisions :

Le questionnaire était à renvoyer à Alain Bernard, par voie électronique ou postale.

\section{NOTES}

1. Pour des raisons bien claires il ne nous est pas permis d'exploiter nominativement les informations recueillies par ce biais. Les informations qui sont exploitées dans ce texte, quand elles sont nominatives, l'ont été en général avec l'accord des personnes concernées, sauf oubli de notre part.

2. Même à ce niveau nous manquons pour l'instant de données pour examiner quelles sont les exigences des programmes, d'une part, les pratiques des enseignants comme des formateurs d'autre part. 
3. Le budget des IREM dépendait jusqu'à il y a peu directement du ministère de l'éducation nationale. Les efforts de décentralisation néanmoins ont conduit à faire dépendre leur budget des universités auxquelles ils sont rattachés, ce qui est susceptible de fragiliser leur position.

4. Les mêmes problèmes se posent a priori pour le travail de publication de brochures, de textes pédagogiques ou de ressources numérisées (éventuellement mises en réseau), qui est une partie parfois importante de l'activité d'un IUFM et n'est pas toujours dissociable d'une activité authentique de recherche. Malheureusement les questionnaires sont très peu informatifs à ce sujet, la question du lien recherche-publication ayant été mal posée.

5. Rappelons que la formation des enseignants au niveau universitaire relève d'organismes spécifiques, les CIES (Centre d'Initiation à l'Enseignement Supérieur).

6. Voir l'article de P. Savaton dans ce même numéro présentant les résultats de son enquête sur les formations en IUFM.

7. En l'occurrence d'une historienne : quelques jours avant le début des journées de mai, Muriel Guedj venait d'être recrutée à l'IUFM de Montpellier, sur un profil didactique et histoire des sciences.

8. Un travail de sauvegarde du patrimoine scientifique de l'UBO a été initié en février 2005 par SL en lien avec la Mission Nationale pilotée par D. Thoulouze et C. Cuenca (CNAM). Il concerne les instruments scientifiques pour la recherche et l'enseignement scientifique ainsi que la documentation (technique, scientifique, pédagogique) et s'inscrit dans un projet de recherche en HST sur la production scientifique en physique d'une université créée en 1964. La collection d'instruments pour TP de Physique associée à des cahiers d'étudiants et la documentation pédagogique permet d'envisager une analyse critique de l'évolution des contenus à montrer, travailler dans ces TP.

9. Notre collègue Sébastien Soubiran a entrepris l'examen d'un corpus scientifique en coopération avec une enseignante locale - si cette action n'a pas débouché pour l'instant sur une formation d'enseignant à proprement parler, il est évident qu'elle pourrait très facilement le faire, comme toute autre expérience de ce type

10. Voir les travaux de Renaud d'Enfert.

11. Il convient de rappeler que la formation initiale et continue pour les enseignants du second degré n'est présente dans les instituts de formation des maîtres que depuis très peu de temps (à peine plus d'une décennie) si on la compare à la vocation traditionnelle des écoles normales d'instituteurs, auxquels ont succédé les IUFM créés suite à la loi de 1989, qui était de former les instituteurs de l'enseignement primaire.

12. A titre d'exemple : le portail Hist-Sciences-Tech (http://histsciences.univ-paris1.fr/) ; Gallica (http://gallica.bnf.fr/); Le site CNUM du CNAM (http://cnum.cnam.fr/) ; Istituto et Museo di Storia della Scienzia (http://www.imss.fi.it/biblio/indice.html).

\section{RÉSUMÉS}

Cet atelier s'est donné pour objectif de traiter deux questions: (Q1) des recherches en épistémologie et histoire des sciences en tant que telles ont-elles leur place dans un IUFM ? (Q2) un domaine de recherche à initier: la didactique de l'histoire des sciences? Après une présentation d'un premier état des lieux de la recherche en EHST présente dans les IUFM et une explicitation du choix de ces deux questions, on trouvera dans la synthèse les trois points qui se 
sont dégagés quant à la justification des recherches EHST en IUFM ainsi que les aspects polémiques que présente la seconde question.

The aim of the paper is to examine two questions: (Q1) do epistemology and history of sciences researches have a place in the French institutions for teacher training (IUFM)? (Q2) A research field to be initiated : the didactic for history of sciences? After a presentation of a first inventory of the research in the field of epistemology and history of sciences in the IUFM and a explanation why we choose these two questions, one will find in the synthesis the three points which emerged for the justification for such research as well as the polemical point of view that the second question shows.

INDEX

Mots-clés : didactique, épistémologie, formation des maîtres, histoire des sciences, recherche

\section{AUTEURS}

\section{ALAIN BERNARD}

Centre Koyré, IUFM Créteil, alainguy.bernard@wanadoo.fr

\section{SYLVAIN LAUBÉ}

CREAD - Centre François Viète, IUFM de Bretagne, sylvain.laube@bretagne.iufm.fr 